\title{
Estudio de seguimiento del desgaste profesional en relación con factores organizativos en el personal de enfermería de medicina interna.
}

\author{
A survey of the professional weakening in relation \\ with organizational factors in the nursing staff of the \\ internal medicine deparment.
}

\section{$M^{a}$ Carmen Gómez Sánchez \\ $M^{a}$ Carmen Álamo Santos \\ Mercedes Amador Bohórquez \\ Fabiola Ceacero Molina \\ Asunción Mayor Pascual \\ Asunción Muñoz González \\ Montserrat Izquierdo Atienza.}

Enfermeras de Medicina Interna del Hospital Universitario

"12 de Octubre" de Madrid

Correspondencia:

Ma Carmen Gómez Sánchez

Medicina Interna

Hospital Universitario "12 de Octubre"

Avda. Córdoba S/N.

Madrid 28041

91-3908109-10

maila77@ya.com

Resumen

El personal de enfermería es uno de los grupos profesionales más afectados por el Síndrome de burnout, debido a la gran cantidad de estresores diarios, inherentes a su profesión, que debe afrontar.

El objetivo de este estudio fue analizar comparativamente el grado de estrés profesional y satisfacción laboral en un grupo de profesionales de enfermería de Medicina Interna en el año 1998 y 2005, e intentar analizar si existía relación con factores organizativos de la Institución.

En ambos años se distribuyen 107 cuestionarios que incluían el Inventario de Burnout de Maslach (MBI) para medir la incidencia de Burnout y el Cuestionario de Satisfacción Laboral de Warr, Cook y Hall (1979).

Se objetivó un descenso del grado de agotamiento emocional y un aumento del grado de realización personal. El grado de satisfacción laboral de los profesionales en ambos años objeto de estudio fue de moderadamente satisfecho.

El hecho de disminuir la sobrecarga laboral y mejorar la seguridad en el puesto de trabajo podría influir positivamente en la reducción de la sobrecarga emocional.

(Med Segur. Trab 2009; 55 (215): 52-62)

Palabras clave: Estrés Laboral, Satisfacción Laboral, Desgaste Profesional, Enfermeras. 


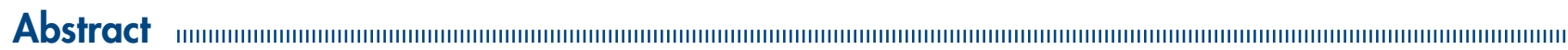

Nursing staff is one of the most affected professional groups the burnout syndrome due to the great quantity of everyday stressful factors which are attached to their profession which nurses have to face up to.

The aim of this survey was to analyze comparatively the professional stress and labour self-satisfaction in a professional nursing staff of the internal medicine department between 1998 and 200 and to try to analyze if there was any relationship with organizational factors of the institution.

107 questionnaires were given out in both years the questionnaires included burnout inventory of Maslach to measure the incidence of burnout and the questionary of laboral self-satisfaction.

The result of the survey was a falling-of the emotional exhaustion degree and an increase of the personal fulfilment degree. satisfied.

The degree of personal self-satisfaction of the nursing staff during these two years was moderately

The fact of decreasing the labour overload and the fact of improving the security in their job could positively have an influence on the lessening of the emotional overload.

(Med Segur Trab. 2009; 55 (215): 52-62)

Key words: Professional stress, labour self-satisfaction, professional weakening, nursing staff. 


\section{INTRODUCCIÓN}

Maslach y Jackson definieron el síndrome de Burnout como una pérdida gradual de preocupación y de todo sentimiento emocional hacia las personas con las que trabajan y que conlleva a un aislamiento o deshumanización. A su vez sentaron las bases para la realización de estudios en diferentes colectividades ya que idearon un instrumento de medida denominado Maslach Burnout Inventory (MBI) ${ }^{1}$.

El Sindrome de Burnout ha sido conceptualizado como un síndrome de agotamiento emocional, despersonalización y baja realización personal en el trabajo, y aparece como consecuencia de un estrés laboral crónico excesivo ${ }^{2}$ que provoca una disminución de la habilidad en el desempeño de la actividad profesional y la aparición de diferentes problemas de salud en quien lo padece ${ }^{3}$.

El Agotamiento Emocional hace referencia a la situación en que la fuerza o el capital emocional se van consumiendo y el profesional siente como se vacía su capacidad de entrega a los demás, tanto desde un nivel personal como psicológico ${ }^{4}$.

La Baja Realización personal en el trabajo se define como la tendencia de los profesionales a evaluarse negativamente de modo que esa evaluación negativa afecta su habilidad en la realización del trabajo y el tipo de relación que establecen con las personas a las que dan servicio. Esta circunstancia provoca que los profesionales se sientan descontentos consigo mismos e insatisfechos con sus resultados laborales 5 .

La Despersonalización o Deshumanización hace referencia a la aparición de sentimientos y actitudes negativas y cínicas hacia las personas destinatarias del trabajo.

La prevalencia del síndrome entre las profesiones catalogadas de riesgo (médicos, enfermeras, profesores, bomberos policías...) va desde un $15 \%$ hasta un $50 \%$ de profesionales afectados ${ }^{6}$.

Dentro de las profesiones sanitarias, la Enfermería representa un grupo de alto riesgo sensible al Sindrome de Burnout, debido a la gran cantidad de estresores diarios inherentes al trabajo clínico que debe afrontar, derivados de la confrontación con el sufrimiento humano, la enfermedad, la invalidez y la muerte ${ }^{7}$. La cantidad de horas diarias que el profesional de enfermería pasa junto al paciente, así como el hecho de ser la persona que habitualmente hace de intermediario ante el paciente, el médico y los familiares son algunas de las razones que hace más vulnerable a este colectivo frente al Síndrome de Burnout.

$\mathrm{Si}$ a estos factores se añaden las características físicas y emocionales de ciertas unidades de trabajo, con pacientes conflictivos, moribundos, duras condiciones de trabajo, fuertes críticas, insuficiente reconocimiento social, imagen devaluada de la profesión, entre otras, conducen a un estado de impotencia e insatisfacción profesional que llevan al desgaste y a una mayor vulnerabilidad frente al Sindrome de Burnout, indicador incuestionable de pérdida de salud.

Las variables organizacionales como la sobrecarga de trabajo, la injusticia, la falta de recompensa por el trabajo desempeñado, los conflictos de valores, la pérdida de cordialidad en el ambiente laboral y la pérdida de control sobre lo que se realiza han sido consideradas como variables desencadenantes o antecedentes y de importancia fundamental en el desarrollo del síndrome, mientras que las personales han sido consideradas como variables que cumplen una función facilitadora o inhibidora en el desarrollo del síndrome, es decir, en la medida que estén o no presentes aumentarán o disminuirán los sentimientos de "sentirse quemado"

Entre las consecuencias más relevantes que repercuten sobre los objetivos y resultados de las organizaciones cabe citar que el síndrome afecta negativamente a la satisfacción laboral y positivamente al deterioro de la calidad de servicio de la organización, la inclinación al absentismo y la propensión al abandono de la organización ${ }^{8}$. 
La satisfacción laboral es uno de los aspectos que ha captado el interés de los psicólogos organizacionales. Al principio dicha atención se centro en los efectos de ésta sobre otras variables como el rendimiento, absentismo, accidentabilidad y el cambio o abandono de la organización ${ }^{9}$. Con posterioridad y desde una orientación en la que los intereses se centraron en torno a la calidad laboral, la investigación ha tomado la satisfacción laboral como una dimensión valiosa en sí misma para la intervención organizacional ${ }^{10}$.

La calidad de vida laboral es un concepto relacionado con las condiciones de trabajo. La no existencia de estrés laboral excesivo, la satisfacción laboral, y la implicación en el trabajo entendida como ausencia de alineación, son los indicadores de calidad de vida laboral que se citan más frecuentemente ${ }^{11}$.

\section{MATERIAL Y MÉTODO}

\section{Objetivo}

Conocer la incidencia de cada una de las tres dimensiones del Síndrome de Burnout y el grado de satisfacción laboral entre el Personal de Enfermería de Medicina Interna del Hospital Universitario 12 de Octubre de Madrid, comparando los datos obtenidos en 1998 y en 2005.

\section{Diseño}

Estudio comparativo con seguimiento del desgaste profesional en relación con factores organizativos en personal de Enfermería del Hospital Universitario 12 de Octubre de Madrid (Diplomados Universitarios en Enfermería y Auxiliares de Enfermería que formaban parte de la plantilla de trabajadores en las unidades de Medicina Interna durante los años 1998 y 2005.

\section{Poblaciones de estudio}

Como muestras poblacionales en ambos años se empleó la totalidad de la plantilla de Personal de Enfermería (DUE y Auxiliares de Enfermería) de Medicina Interna del Hospital Universitario "12 de Octubre" de Madrid. En este hospital existen 3 plantas de Medicina Interna con un total de 132 camas, distribuidas en las plantas 12, 13 y 15 del hospital. En ellas trabajaban 57 enfermeras y 48 Auxiliares de enfermería en ambos años.

\section{Variables}

En ambos estudios se recogieron variables demográficas como la edad, el sexo, el estado civil, el $\mathrm{n}^{\circ}$ de hijos, el nivel de estudios, los años de ejercicio profesional, los años de ejercicio profesional en Medicina Interna, el turno de trabajo, el índice de participación por plantas y la situación laboral (personal fijo, contratado o interino).

Otras variables objeto de estudio fueron las subescalas del síndrome de burnout: agotamiento emocional, despersonalización y baja realización personal.

En relación con el grado de satisfacción laboral, las variables analizadas en ambos estudios fueron: Condiciones físicas de trabajo, libertad de escoger el método de trabajo, los compañeros de trabajo, el reconocimiento por haber realizado un buen trabajo, el jefe inmediato, la cantidad de responsabilidad, el dinero percibido, la oportunidad de emplear las capacidades, relaciones sociales entre la dirección y los trabajadores, las oportunidades de promoción, la forma en que está dirigida la empresa, la atención prestada a las sugerencias, las horas de trabajo, las actividades, la seguridad en el puesto de trabajo.

\section{Recogida de muestras y registro de datos} incluían:

En ambos años se distribuyen 107 cuestionarios autoadministrados y validados que 
- MBI: inventario de Burnout de Maslach1. Evalúa los tres aspectos del Síndrome de Burnout: agotamiento emocional, despersonalización y la disminución de los logros. El MBI fue validado por Maslach y Jackson, y su versión definitiva apareció en 1986.

- Cuestionario de Satisfacción Laboral Warr, Cook y Hall (1979). (Anexo 1)

\section{Análisis estadístico}

Se llevó a cabo un estudio de las interrelaciones de las variables que se pretendían estudiar: subescalas de Burnout e ítems de satisfacción laboral, mediante el paquete estadístico SAS (análisis de correlación entre cada una de las variables). Se realizó la correlación de Spearman, el test de Wilconxon y Chi-cuadrado.

\section{RESULTADOS}

\section{Características de las poblaciones de estudio}

En el grupo de población correspondiente al año 1998, se obtuvo respuesta a los cuestionarios en 71 casos de los 107 distribuidos (66'36\%), el 88'7\% son mujeres y el 13 '3\% hombres. El rango de edad fue de 24 a 50 años, la media fue de 33'8 con una desviación estándar de \pm 7’0 años.

En el grupo de población correspondiente al año 2005, se obtuvo respuesta de 61 cuestionarios de un total de 107 cuestionarios distribuidos (57,01\%), el 92'8\% eran mujeres y el 7’15\% hombres. El rango de edad fue de 21 a 54 años, siendo la media de edad de 36 '55 con una desviación estándar de \pm 10 '9 años.

El índice de participación por plantas en la cumplimentación de los cuestionarios en el año 1998 fue del 42,25\% en la planta 12, el 26,76\% en la planta 13 y un 30,99\% en la planta 15. En el año 2005 el índice de participación por plantas fue del 50,82\% en la planta 12 , del $31,15 \%$ en la planta 13 y del $18,03 \%$ en la planta 15 .

Estado civil. En el año 1998 el 48,2 \% de los trabajadores eran solteros, el 5,4\% eran casados y el 1,8\% vivían en pareja. En el año 2005 el 38,98\% eran trabajadores solteros, el 5,08\% eran divorciados, casados el 2,54\% y vivían en pareja el 3,39\%.

Número de hijos. En el año 1998 el 54,55\% de estos trabajadores no tenía hijos, el 41,55\% tenía un hijo, el 27,27\% tenía 2 hijos, el 9,09\% tenía 3 hijos y el 4,5\% tenía 5 hijos. En el año 2005 el 51,67\% no tenía hijos, el 1\% tenía 1 hijo, el 1,67\% tenía 3 hijos y el 1,67\% tenía 4 hijos.

Respecto al nivel de estudios de estos profesionales, en el año 1998 el 67,86\% eran Diplomados en Enfermería, el 12,5 \% tenían el nivel FP1 y el 5,36\% FP2. En el año 2005, el $66,67 \%$ de las personas que completaron el cuestionario eran Diplomados en Enfermería, el 23,33\% habían cursado estudios de FP1 y el 10\% FP2.

Categoría profesional. La participación de Enfermeras en el año 1998 fue del 66,09\% y del 33,97\% de Auxiliares de Enfermería en 1998, en el año 2005 el 65\% de los participantes en el estudio se trataba de Enfermeras y el 35\% eran Auxiliares de Enfermería.

Turno de trabajo. En el año 1998 el 68,52\% de los profesionales que participaron en el estudio pertenecían al turno de mañana y el 31,48\% al turno de tarde. En el año 2005 el $61,67 \%$ correspondía al turno de mañana y el 38,37\% al turno de tarde. En nuestro hospital no existe turno de noches fijas, las noches las realizan de forma rotatoria el personal del turno de mañana y del turno de tarde.

Respecto a la situación laboral en el año 1998 un 1,79\% era personal fijo, el 42,86\% tenían un contrato de interinidad y el 53,57\% era personal eventual. En el año 2005 el 33,30\% era personal fijo, el 11,67\% eran interinos y el 55,03\% tenía un contrato eventual.

Años de ejercicio profesional. En el año 1998 la media en meses de ejercicio profesional era de 127,6 +/- 75,7. La media en años de trabajo era de 10,5. En los resultados 
obtenidos en el año 2005 la media en meses de ejercicio profesional era de 139,95 +/93,4. La media en años de ejercicio profesional era de 11,7.

Años de ejercicio profesional en Medicina Interna. El tiempo de ejercicio profesional en el servicio de Medicina Interna en 1998 fue de 102,2 meses +/- 62,04. Media en años de ejercicio profesional 8,5. En el año 2005 la media de ejercicio profesional en meses de estos trabajadores en unidades de Medicina Interna fue de 8,87 +/- 79,09. Media en años de 7,15.

Comparando todos los datos demográficos y laborales de los dos grupos estudiados correspondientes a los años 1998 y al 2005, no encontramos significación estadística para ninguna de las variables demográficas y profesionales estudiadas, salvo de la situación laboral (ser personal fijo o contratado) con una $p=0^{\prime} 0322$, lo que nos indica que las dos poblaciones estudiadas pueden considerarse homogéneas en cuanto a estas variables estudiadas y en consecuencia, susceptibles de ser comparadas respecto a las variables objeto de estudio de Burnout y de la satisfacción laboral.

\section{Resultados de las variables de estudio}

Desde una perspectiva psicosocial el síndrome de Burnout se presenta cuando los síntomas son bajos en los niveles de realización personal en el trabajo y hay altos niveles de agotamiento emocional y de despersonalización ${ }^{11}$.

Tabla 1: Valores medios de síndrome de desgaste profesional en el ámbito sanitario. Adaptada de la escala de Maslach a la población española por Moreno, Oliver y Aragoneses ${ }^{7}$.

\begin{tabular}{lcccc}
\hline Ámbito Sanitario & Media & Bajo & Medio & Alto \\
\hline Agotamiento Emocional & $22,19+/-9,3$ & $<18$ & $19-26$ & $>27$ \\
Despersonalización & $7,21+/-5,22$ & $<5$ & $6-9$ & $>10$ \\
Realización Personal & $36,53+/-7,34$ & $<33$ & $34-39$ & $>40$ \\
\hline
\end{tabular}

Respecto a los resultados obtenidos de la variable respuesta de las subescalas de burnout en nuestro estudio, en el año 1998 la media de agotamiento emocional fue de 22,49 con una desviación estándar de 11,36 que corresponde a un grado medio de agotamiento emocional. En el año 2005 la media de esta subescala fue de 19,63 con una sd de 10,14, que supone un nivel medio-bajo de agotamiento emocional.

En cuanto al grado de despersonalización o deshumanización, en el año 1998 encontramos una media de 7,86 con una Sd de 5,75, que corresponde a un grado medio. En el año 2005 la media de la variable despersonalización fue de 6,69 con una Sd de 5,13 que corresponde también a un grado medio.

Con respecto a la subescala realización personal en el año 1998 la media fue de 36,27 con una desviación estándar de 7,27, que correspondía a un grado medio. En el año 2005 la media fue de 39,67 con una Sd de 7,22 que supone un grado medio-alto de falta de realización personal.

Tabla 2. Resultados de las subescalas del síndrome de Burnout obtenidos en otros estudios realizados en el ámbito sanitario.

\begin{tabular}{|c|c|c|c|}
\hline Otros Estudios & Despersonalización & Agotamiento emocional & Realización personal \\
\hline Adali $E^{12}$ & 6,43 & 18,36 & 34,62 \\
\hline Atance Martinez $\mathrm{JC}^{13}$ & 11,28 & 14,84 & 21,32 \\
\hline Albadalejo $R^{14}$ & 8,37 & 19,61 & 20,80 \\
\hline Pera $G^{15}$ & 6,83 & 18,59 & 40,58 \\
\hline
\end{tabular}


Analizados los resultados de la encuesta de satisfacción laboral mediante el Cuestionario de Satisfacción Laboral de Warr, Cook y Hall (1979) los resultados obtenidos fueron que en el año 1998, los profesionales de enfermería estaban muy insatisfechos con el sueldo que percibían, las relaciones con la dirección del hospital, las posibilidades de promoción, la forma en que estaba dirigida la empresa, y la seguridad en el puesto de trabajo, moderadamente insatisfechos con las condiciones de trabajo, no estaban seguros del grado de reconocimiento por su trabajo, de la oportunidad para emplear sus capacidades, ni del grado de interés que se prestaban a sus sugerencias, se encontraban moderadamente satisfechos con el método de trabajo, sus colegas, su jefe inmediato, el grado de responsabilidad que le daban, la cantidad de horas trabajadas y las actividades que se llevaban a cabo en su unidad. La valoración general de los trabajadores del estudio en el año 1998 era de moderadamente satisfechos. (Gráfico 1 y 2). Tabla 3.

En el año 2005 los trabajadores sanitarios objetos de estudio se manifestaban moderadamente insatisfechos con las condiciones de trabajo, el dinero que percibían, las relaciones con la dirección, las posibilidades de promoción, la forma en la que estaba dirigida la empresa, y la seguridad en el puesto de trabajo, moderadamente satisfechos con el método de trabajo, el grado de reconocimiento por su trabajo, su jefe inmediato, el grado de responsabilidad que le daban, la atención que se prestaba a su capacidad, las actividades que se realizaban en su unidad y muy satisfechos con sus compañeros de trabajo. La valoración general de los participantes en el estudio en el año 2005 fue de moderadamente satisfechos. (Gráfico 1 y 2). Tabla 3.

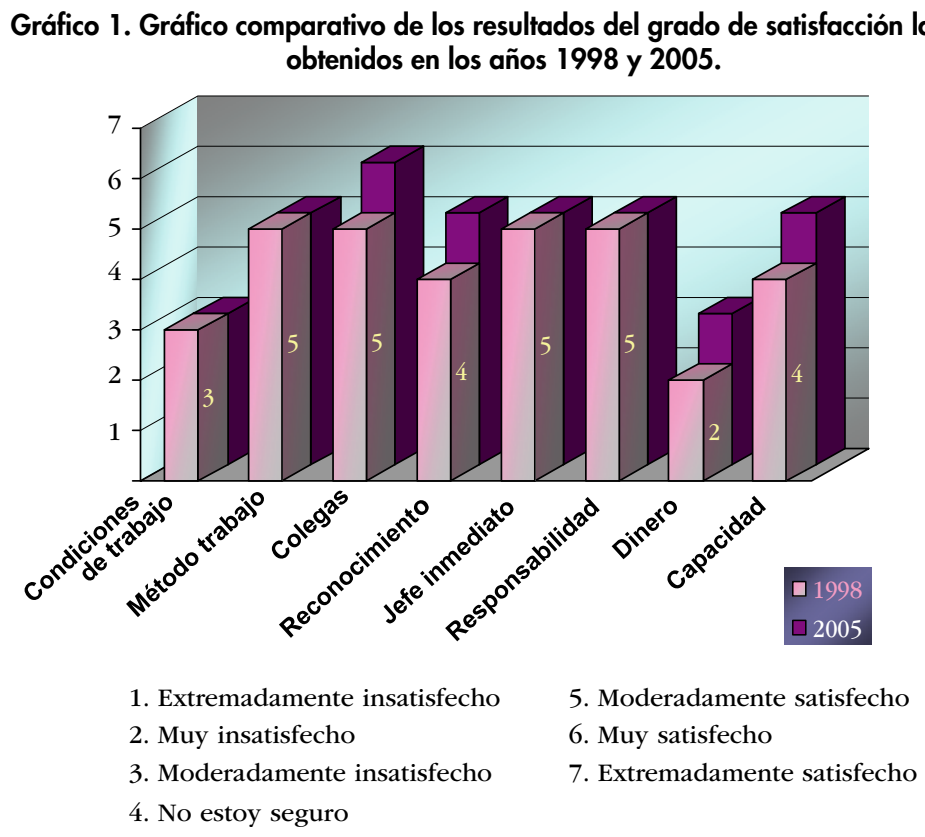

Comparando los resultados de las variables de satisfacción laboral de los años 1998 y 2005 mediante el test de Wilcoxon se encontró significación estadística en: La libertad de escoger el método de trabajo: $\mathrm{p}=0,0284$. La relación con los colegas: $\mathrm{p}=0,0012$. El dinero percibido: $\mathrm{p}<0,0001$. Las relaciones con la dirección: $\mathrm{p}=0,0025$. Las oportunidades de promoción: $\mathrm{p}=0,0005$.

No se encontró significación estadística al comparar las condiciones físicas de trabajo, el reconocimiento por haber realizado un buen trabajo, el jefe inmediato, la cantidad de responsabilidad, la oportunidad de emplear las capacidades, la forma en que está dirigida la empresa, la atención prestada a las sugerencias, las horas de trabajo, las actividades realizadas, la seguridad en el puesto de trabajo. 
Gráfico 2. Gráfico comparativo de los resultados del grado de satisfacción laboral obtenidos en los años 1998 y 2005.

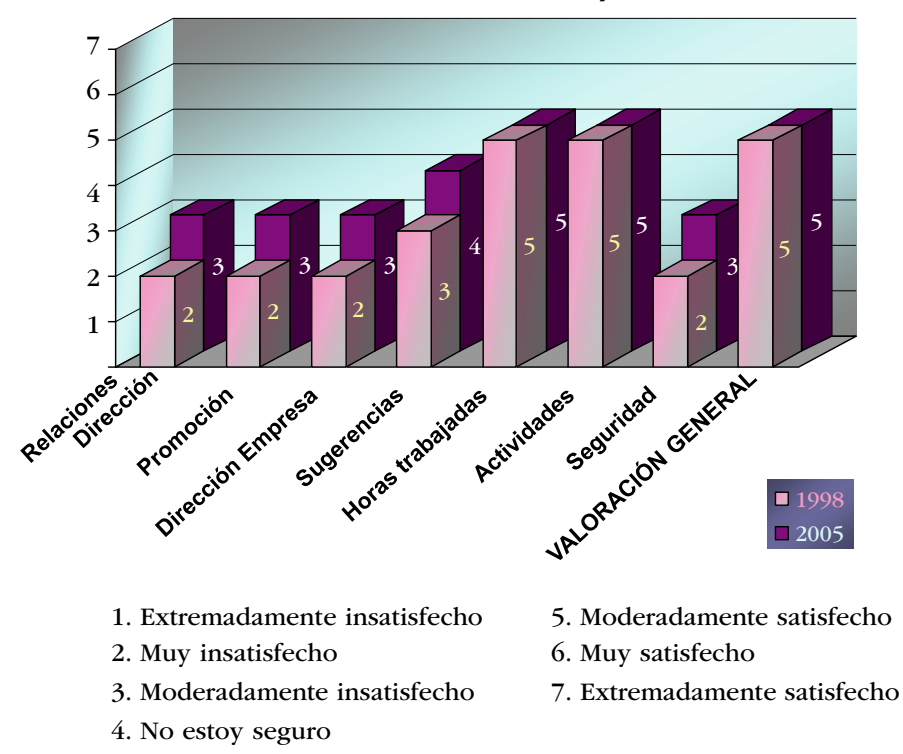

Tabla 3: Resultados del Grado de satisfacción laboral en los años 1998 y 2005

\section{SATISFACCIÓN LABORAL}

Condiciones de trabajo

Método de trabajo

Relación con los colegas

Reconocimiento por trabajo bien hecho

Jefe inmediato

Grado de responsabilidad

Dinero percibido

Oportunidad emplear las capacidades

Relaciones con la dirección

Oportunidades de Promoción

Forma de Dirección de la empresa

Atención prestada a sus Sugerencias

Las Horas de trabajo

Actividades realizadas

Seguridad en el puesto de trabajo

VALORACIÓN GENERAL
Año 1998

Moderadamente insatisfecho

Moderadamente satisfecho

Moderadamente satisfecho

No estoy seguro

Moderadamente satisfecho

Moderadamente satisfecho

Muy insatisfecho

No estoy seguro

Muy insatisfecho

Muy insatisfecho

Muy insatisfecho

Moderadamente insatisfecho

Moderadamente satisfecho

Moderadamente satisfecho

Muy insatisfecho

Moderadamente satisfecho
Año 2005

Moderadamente insatisfecho

Moderadamente satisfecho

Muy satisfecho

Moderadamente satisfecho

Moderadamente satisfecho

Moderadamente satisfecho

Moderadamente insatisfecho

Moderadamente satisfecho

Moderadamente insatisfecho

Moderadamente insatisfecho

Moderadamente insatisfecho

No estoy seguro

Moderadamente satisfecho

Moderadamente satisfecho

Moderadamente insatisfecho

Moderadamente satisfecho

\section{DISCUSIÓN}

En el año 1998 existía un grado medio de agotamiento emocional y medio-bajo en el año 2005 en el personal de Enfermería de Medicina Interna del Hospital Universitario 12 de Octubre de Madrid. En ambos años había un grado medio de despersonalización o deshumanización entre los profesionales y en cuanto a la realización personal había un 
grado medio en el año 1998 y medio-alto en el 2005. En el año 2005 disminuyó el agotamiento emocional, se mantenía estable el grado de despersonalización y mejoró la realización personal. Hay que tener en cuenta que puntuaciones altas en el agotamiento emocional y despersonalización y bajas en realización personal definen el Síndrome de Burnout.

Respecto a las variables demográficas y laborales (edad, sexo, estado civil, número de hijos, nivel de estudios, categoría profesional, años de ejercicio profesional, años de trabajo en Medicina Interna) no se encontró significación estadística en ninguna de ellas salvo en la situación laboral, ser personal fijo o contratado ( $p=$ O’0322)

El grado de satisfacción laboral en el año 2005, había mejorado con respecto a los resultados obtenidos en 1998, se mantenía sin variaciones en las condiciones físicas de trabajo, la libertad de escoger el método de trabajo, el jefe inmediato, la cantidad de responsabilidad, las horas de trabajo y la valoración general. (Gráfica 1 y 2)

Se detectó una mejoría en cuanto a la relación con los colegas de trabajo, el reconocimiento por un trabajo bien hecho, el dinero percibido, la oportunidad para emplear sus capacidades, las relaciones sociales entre dirección y trabajadores de la empresa, las oportunidades de promoción, la forma en que estaba dirigida la empresa, la atención que prestaban a sus sugerencias, y la seguridad en el puesto de trabajo. (Gráfica 1 y 2)

La percepción general fue de mejora. En ningún caso se objetivó descenso del grado de satisfacción laboral.

Los cambios apreciables que se habían producido en el Hospital entre estos dos años objeto de estudio y que es posible que influyeran en el grado de satisfacción laboral de los trabajadores fueron entre otros, que en el año 1998, una inmensa mayoría de profesionales interinos de nuestro hospital obtuvieron plaza en propiedad por concurso-oposición del INSALUD. (Año 1998: Personal fijo: 1,79 \%, personal interino: $42,86 \%$ y personal eventual: 53,57 \%. Año 2005: Personal fijo: 33,30 \%, personal interino: 11,67 \%. Personal eventual: 55,03 \%), así como un cambio de la Dirección de Enfermería del hospital. Las relaciones con la Dirección y la forma en que estaba dirigida la empresa obtuvieron un grado de satisfacción laboral del personal de Enfermería de muy insatisfecho en el año 1998 y moderadamente insatisfecho en el año 2005.

En el año 2005 se disponía de un pool de trabajadores que cubría las incidencias de personal, lo que contribuyó a disminuir la sobrecarga de trabajo y posiblemente a aumentar el grado de satisfacción laboral. El hecho de disminuir la sobrecarga de trabajo, podría influir positivamente en la reducción de la sobrecarga emocional.

Los resultado de la investigación dejan entrever que a medida que aumentó la satisfacción laboral del personal de enfermería en cuanto a los recursos organizativos, se redujo el desgaste profesional ya que los datos obtenidos indicaban que disminuyó el agotamiento emocional y aumentó la realización personal de los trabajadores objeto de estudio, manteniéndose en un grado medio la despersonalización o deshumanización.

\section{AGRADECIMIENTOS}

Nuestro más sincero agradecimiento al Dr José Carlos Mingote Adán Coordinador Psiquiatra del PAIPSE y al Dr Agustín Gómez de la Cámara y todo el equipo de la Unidad de Epidemiología Clínica. Unidad de Investigación del Hospital Universitario "12 de Octubre" de Madrid. 


\section{BIBLIOGRAFÍA}

1. Maslach C, Jackson S. Maslach Burnout Inventory. Palo Alto: Consulting Psychologist; 1986

2. Schaufeli, W.B., Maslach, C. y Marek, T. Profesional burnout: Recent developments in theory and research. Washington DC: Taylor \& Francis; 1993

3. Maslach C, Schaufeli WB, Leiter MP (2001): "Job burnout", Annual Review of Psychology, 2001; 52: $397-422$.

4. Seisdedos N. Manual MBI Inventario Burnout de Maslach, Síndrome del quemado por estrés laboral asistencial. Madrid: Tea Ediciones; 1997.

5. Gil Monte P. El Síndrome de quemarse por el trabajo (Burnout). Madrid: Pirámide; 2005.

6. Apellániz González A, Pascual Izaola A. Enfermedades laborales emergentes: el burnout. Prevención: Revista Técnica de seguridad y salud laborales.2005; 172: 26-34.

7. Mingote Adán JC. Síndrome burnout. Monogr Psiquiatr .1997; IX (5).

8. Gil-Monte PR, Peiró JM. Desgaste psíquico en el trabajo: el Síndrome de quemarse. Madrid: Síntesis; 1997.

9. Algag R J, Brief AP. (1978). Examination of alternative models of job satisfaction. Human Relations. 1978; 31: 91-98.

10. Quinn RP, Gonzales T A. A consumer's guide to job satisfaction measures. In Abrahamson T, Tittle CK, Cohen L (Eds). Handbook of vocational Education Evaluation. Sage Publications: London; 1979.

11. Aycan Z, Kanungo R.N. Cross-cultural Industrail and Organizational Psychology: A critical appraisal of the field and future directions. In: Anderson N, Ones DS, Sinangil HK, Viswesvaran C, editors. Handboock of Industrial, Work and Organizational Psychology. Londres: Sage; 2001: 385-408.

12. Gil Monte PR. El síndrome de quemarse por el trabajo (Síndrome de Burnout) en profesionales de enfermería. Revista Eletrônica InterAção Psy.2003 1, 19-33

13. Aldalie E, Priami M, Evagelou H, Mougia V, Ifanti M, Alevizopoulos G. Síndrome del Quemado en el personal de enfermería psiquiátrica de hospitales griegos. Eur. J. Psychiat. 2003; 17 (3): 161-70

14. Atance Martinez JC. Aspectos epidemiológicos del síndrome de Burnout en personal sanitario. Rev. Esp. Salud Pública. 1997; 71(3): 293-303.

15. Albaladejo R, Villanueva R, Ortega P, Astasio P, Calle ME, Domínguez V. Síndrome de Burnout en el personal de enfermería de un hospital de Madrid. Rev. Esp. Salud Pública. 2004; 78(4): 505-516.

16. Pera G, Serra-Prat M. Prevalencia del síndrome del quemado y estudio de los factores asociados en los trabajadores de un hospital comarcal. Gac Sanit. 200216 (6): 480-486.

17. Mardarás E. La prevención primaria del riesgo psicosocial. Prevención: Revista Técnica de seguridad y salud laborales.2005; 172: 46-71. 


\section{ANEXO 1}

\section{SATISFACCIÓN EN EL TRABAJO. (WARR, COOK Y WALL, 1979)}

Introducción: Los siguientes puntos muestran varios aspectos de su trabajo.

Diga cómo está usted de satisfecho con cada una de estas características en su trabajo

1. Extremadamente insatisfecho

2. Muy insatisfecho.

3. Moderadamente insatisfecho.

4. No estoy seguro.

5. Moderadamente satisfecho.

6. Muy satisfecho.

7. Extremadamente satisfecho.

Las condiciones físicas de trabajo

La libertad de escoger su propio método de trabajo

Sus colegas de trabajo

El reconocimiento por haber realizado un buen trabajo

Su jefe inmediato

La cantidad de responsabilidad que le dan

El dinero que le pagan

La oportunidad para emplear sus capacidades

Relaciones sociales entre dirección y

trabajadores en su empresa

Sus oportunidades de promoción

La forma en que está dirigida su empresa

La atención que prestan a sus sugerencias

Sus horas de trabajo

Las diferentes actividades a realizar en su trabajo

La seguridad en el puesto de trabajo

Ahora, considerando todo, ¿cómo se encuentra en su trabajo en general?
1

$2-3$

$\begin{array}{llllll}2 & 3 & 4 & 5 & 6 & 7\end{array}$

$\begin{array}{llllll}2 & 3 & 4 & 5 & 6 & 7\end{array}$

$\begin{array}{lllllll}1 & 2 & 3 & 4 & 5 & 6 & 7\end{array}$

$\begin{array}{lllllll}1 & 2 & 3 & 4 & 5 & 6 & 7\end{array}$

$\begin{array}{lllllll}1 & 2 & 3 & 4 & 5 & 6 & 7\end{array}$

$\begin{array}{lllllll}1 & 2 & 3 & 4 & 5 & 6 & 7\end{array}$

$\begin{array}{lllllll}1 & 2 & 3 & 4 & 5 & 6 & 7\end{array}$

$\begin{array}{lllllll}1 & 2 & 3 & 4 & 5 & 6 & 7\end{array}$

$\begin{array}{lllllll}1 & 2 & 3 & 4 & 5 & 6 & 7\end{array}$

$\begin{array}{lllllll}1 & 2 & 3 & 4 & 5 & 6 & 7\end{array}$

$\begin{array}{lllllll}1 & 2 & 3 & 4 & 5 & 6 & 7\end{array}$

$\begin{array}{lllllll}1 & 2 & 3 & 4 & 5 & 6 & 7\end{array}$

$\begin{array}{lllllll}1 & 2 & 3 & 4 & 5 & 6 & 7\end{array}$

$\begin{array}{lllllll}1 & 2 & 3 & 4 & 5 & 6 & 7\end{array}$

$\begin{array}{lllllll}1 & 2 & 3 & 4 & 5 & 6 & 7\end{array}$

